\title{
Antibiotic and chemotherapeutic enhanced three-dimensional printer filaments and constructs for biomedical applications
}

This article was published in the following Dove Press journal:

International Journal of Nanomedicine

9 January 2015

Number of times this article has been viewed

\author{
Jeffery A Weisman' \\ James C Nicholson ${ }^{2}$ \\ Karthik Tappa' \\ UdayaBhanu \\ Jammalamadaka' \\ Chester G Wilson² \\ David K Mills ${ }^{1,3}$ \\ 'Center for Biomedical Engineering \\ and Rehabilitation Science, \\ ${ }^{2}$ Nanosystems Engineering, ${ }^{3}$ School \\ of Biological Sciences, Louisiana \\ Technical University, Ruston, LA, USA
}

Correspondence: David K Mills

I Adams Blvd, School of Biological

Sciences/CBERS, Louisiana Tech

University, Ruston, LA 7I272, USA

Tel + I 8002572640

Fax + I 8002574574

Email dkmills@latech.edu

\begin{abstract}
Three-dimensional (3D) printing and additive manufacturing holds potential for highly personalized medicine, and its introduction into clinical medicine will have many implications for patient care. This paper demonstrates the first application of $3 \mathrm{D}$ printing as a method for the potential sustained delivery of antibiotic and chemotherapeutic drugs from constructs for patient treatment. Our design is focused on the on-demand production of anti-infective and chemotherapeutic filaments that can be used to create discs, beads, catheters, or any medical construct using a 3D printing system. The design parameters for this project were to create a system that could be modularly loaded with bioactive agents. All 3D-printed constructs were loaded with either gentamicin or methotrexate and were optimized for efficient and extended antibacterial and cancer growth-inhibiting cytostatic activity. Preliminary results demonstrate that combining gentamicin and methotrexate with polylactic acid forms a composite possessing a superior combination of strength, versatility, and enhanced drug delivery. Antibacterial effects and a reduction in proliferation of osteosarcoma cells were observed with all constructs, attesting to the technical and clinical viability of our composites. In this study, 3D constructs were loaded with gentamicin and methotrexate, but the method can be extended to many other drugs. This method could permit clinicians to provide customized and tailored treatment that allows patientspecific treatment of disease and has significant potential for use as a tunable drug delivery system with sustained-release capacity for an array of biomedical applications.
\end{abstract}

Keywords: three-dimensional printing, polymers, antibacterial, drug delivery, medical device

\section{Introduction}

Developed in the early 1990s by Sachs et al three-dimensional (3D) printing technology at that time was a powder-based fabrication method that was focused towards rapid tool production using metals and ceramics. ${ }^{1,2}$ A typical printing set-up produces a 3D model based on a digital file through an additive process that lays down successive layers with the capability of producing almost any shape. ${ }^{3-5} 3 \mathrm{D}$ printing is best known for creating plastic prototypes and structures rapidly and cheaply, and with a fine degree of resolution. The most common method of fabrication is known as fused deposition modeling, a term that has recently become nearly synonymous with $3 \mathrm{D}$ printing. For this method, a plastic filament is melted and laid down in a layer-by-layer process to build a construct. Developments over the last 10 years have shown that 3D printing has found its place in medicine, nanotechnology, and tissue engineering, thus paving the way for the rapid printing of medicines, artificial devices, prosthetics, and even human tissue. ${ }^{6,7}$ Currently, there is an intense research effort focused on the application of 3D printing 
for development of blood vessels and bioengineered tissues, as well as production of functional biomedical materials and devices for dental and orthopedic applications. ${ }^{8-14}$

Osteomyelitis is a bone infection that can affect both the young and the elderly. ${ }^{14}$ It is generally treated with local or systemic antibiotic therapy and often requires multiple surgical interventions. Osteomyelitis is difficult to treat, and for the patient who is unresponsive to antibiotic therapy, it can lead to amputation or even death. ${ }^{15}$ Chronic osteomyelitis often requires surgical debridement or local antibiotic treatment. ${ }^{15,16}$ Implanted antibiotic-impregnated beads have numerous advantages over systemic therapy. These include ease of placement, decreased systemic toxicity, reduced hospital stay, and less patient cost. ${ }^{16-18}$ While effective, the disadvantages of currently used non-biodegradable polymethyl-methacrylate beads include low biocompatibility, poor antibiotic release, need for surgical removal, and cytotoxic effects due to thermal damage during polymethyl-methacrylate polymerization. ${ }^{17-20} \mathrm{~A}$ biodegradable antibiotic carrier would offer many advantages. There is no need for secondary surgery, and biodegradable carriers are gradually replaced by ingrowing tissue and may even support new bone growth. ${ }^{21,22}$ Additionally, further antibiotic release during degradation could increase antimicrobial efficacy when compared with non-biodegradable carriers. ${ }^{23-25}$

Systemic delivery of antibiotics affects the intended site as well as surrounding uninfected tissues, and for some patients, carries risks of hepatocytotoxicity and nephrotoxicity, and has the potential to increase antibiotic resistance. It is clinically advantageous to have direct treatment without unnecessary and widespread harmful effects. A key goal in this field is to combine the key features of $3 \mathrm{D}$ printing (ability to make complex geometries, speed, tunability) into a system that enables on-demand, customized, and patient-specific antibiotic treatment, and has the potential to address many of the disadvantages with current antibiotic carrier systems.

In the present work, a new class of bioactive $3 \mathrm{D}$ printing filaments using gentamicin sulfate and methotrexate (MTX) was created. It was found that these compounds retained their effective antimicrobial or cytostatic properties throughout the manufacturing process despite the heat required for this method of fabrication.

\section{Materials and methods Materials}

All treatment compounds used in this study were purchased from Sigma-Aldrich (St Louis, MO, USA), including gentamicin sulfate (GS) and MTX. The polylactic acid (PLA) pellets used for the printing media were obtained from
NatureWorks, LLC (Minnetonka, MN, USA). For bacterial culture, Escherichia coli ATCC ${ }^{\circledR} 11775$ Vitroids $^{\mathrm{TM}} 1,000$ colony-forming units were purchased from Sigma-Aldrich. Bacterial culture supplies, $100 \mathrm{~mm}$ Mueller Hinton agar plates, and Mueller Hinton liquid broth $1 \mathrm{~L}$ were purchased from Fischer Scientific (Hampton, NH, USA). Cell culture plates and other laboratory plastics were purchased from MidSci (St Louis, MO, USA). Dulbecco's phosphate-buffered saline, Dulbecco's Modified Eagle's Medium, fetal bovine serum, penicillin-streptomycin-amphotericin antibiotics, and a live/dead viability/cytotoxicity kit were obtained from Life Technologies (Carlsbad, CA, USA). KJLC 705 silicone oil used for coating the pellets prior to extrusion was purchased from Kurt J Lesker Company (Jefferson Hills, PA, USA). The 3D printing set-up consisted of an extruder purchased from ExtrusionBot, LLC (Phoenix, AZ, USA) and a MakerBot 2X 3D printer (Brooklyn, NY, USA).

\section{Preparation of PLA pellets}

Batches of PLA pellets ( $20 \mathrm{~g})$ were added to a $50 \mathrm{~mL}$ sterile plastic tube. KJLC 705 silicone oil $(20 \mu \mathrm{L})$ was added and the tube was vortexed until the pellets were consistently coated. The pellets were then placed in a new $50 \mathrm{~mL}$ sterile plastic tube to ensure minimal losses to oil surface coating of the initial tube. To make $1 \mathrm{wt} \%$ or $2.5 \mathrm{wt} \%$ coatings, respectively, $200 \mathrm{mg}$ or $500 \mathrm{mg}$ of GS or MTX powder was then added to the pellets, which were then vortexed (Figures 1 and 2).

\section{Extrusion of filaments}

The ExtrusionBot filament extruder was used for creation of filaments and consisted of a vertical column with a hopper leading directly into a melt chamber consisting of an auger housed in a heated pipe. The metal die at the extrusion point had a bore of $1.75 \mathrm{~mm}$ in diameter. The temperature is adjusted through a control panel on the front. The typical extrusion temperature for PLA pellets is around $170^{\circ} \mathrm{C}$, depending on ambient temperature and humidity conditions. It should be noted the degradation temperature of GS and MTX are different, and the extrusion temperatures for each of these processes were modified to ensure minimal degradation of the additives within the filament. For GS, a temperature of $175^{\circ} \mathrm{C}$ was used, while MTX required a lower extrusion temperature of $160^{\circ} \mathrm{C}$.

\section{D printing}

Once the filaments were extruded, $5 \mathrm{~mm} 1 \mathrm{wt} \%$ and $2.5 \mathrm{wt} \%$ GS discs were $3 \mathrm{D}$-printed on the MakerBot $2 \mathrm{X}$ at $220^{\circ} \mathrm{C}$. The layer printing height was set to a $300 \mu \mathrm{m}$ resolution with the 


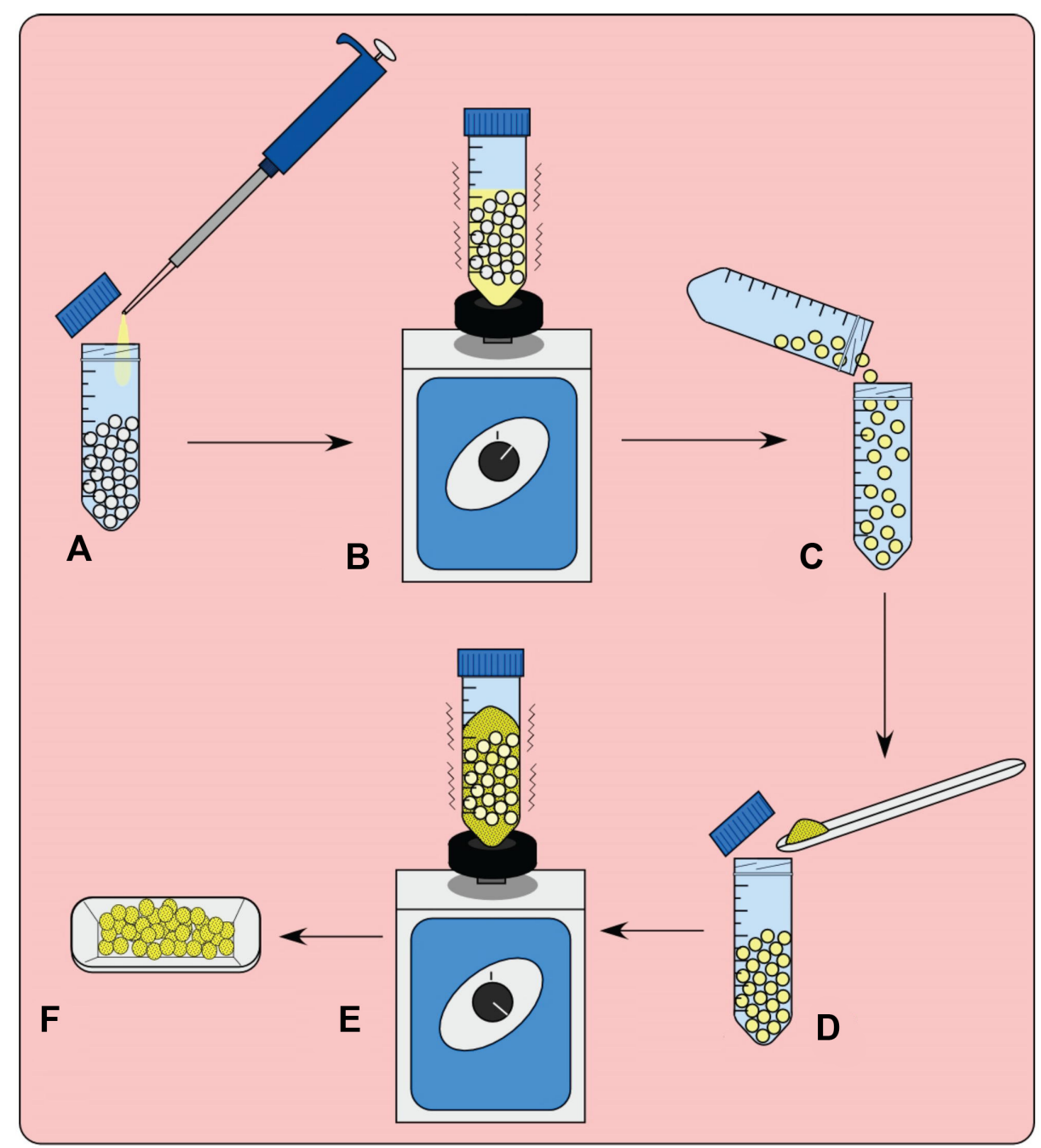

Figure I Method of polylactic acid pellet coating process.

Notes: (A) Coating oil is added. (B) Tube is vortexed. (C) Pellets are transferred to a new tube. (D) Powder is added. (E) Tube is vortexed. (F) Coated beads are removed.

in-fill setting on the software at $100 \%$. It should be noted that while the software for FDM printers could go up to $100 \%$ fill, there are still pores present in FDM-printed constructs. The SolidWorks computer software modeling program (Dassault Systèmes SolidWorks Corporation, Waltham, MA, USA) was used to design the $3 \mathrm{D}$ print files. The resulting STL file generated was used to dictate the construct dimensions to the printer through MakerWare.

\section{Imaging}

Filaments were imaged using field emission scanning electron microscopy (model S4800, Hitachi, Schaumburg, IL, USA) at $35 \times, 2,000 \times$, and $15,000 \times$ magnifications. Bacterial plates and cultures were photographed with a Canon camera. The kill zone of the agar diffusion test was measured with a caliper at three different locations for each filament or disc.

\section{Cultures}

\section{Bacterial cultures}

All the samples were tested for antimicrobial activity by two methods, ie, agar diffusion and liquid nutrient broth. In addition to the above groups, negative controls were also tested for antibiotic activity. A set of positive controls was used and included antibiotic powder of the respective categories. Plain PLA samples and heated antibiotic powders were taken as negative controls. Escherichia coli (E. coli) vitroids were used to raise bacterial colonies, and one colony was picked to make $0.5 \mathrm{McF}$ arland standard bacterial suspensions.

\section{Agar diffusion assays}

In accordance with ISO standard 17025 for measuring bacterial zones of inhibition, Mueller Hinton agar was taken and to this $50 \mu \mathrm{L}$ of bacterial culture was added. Samples were 

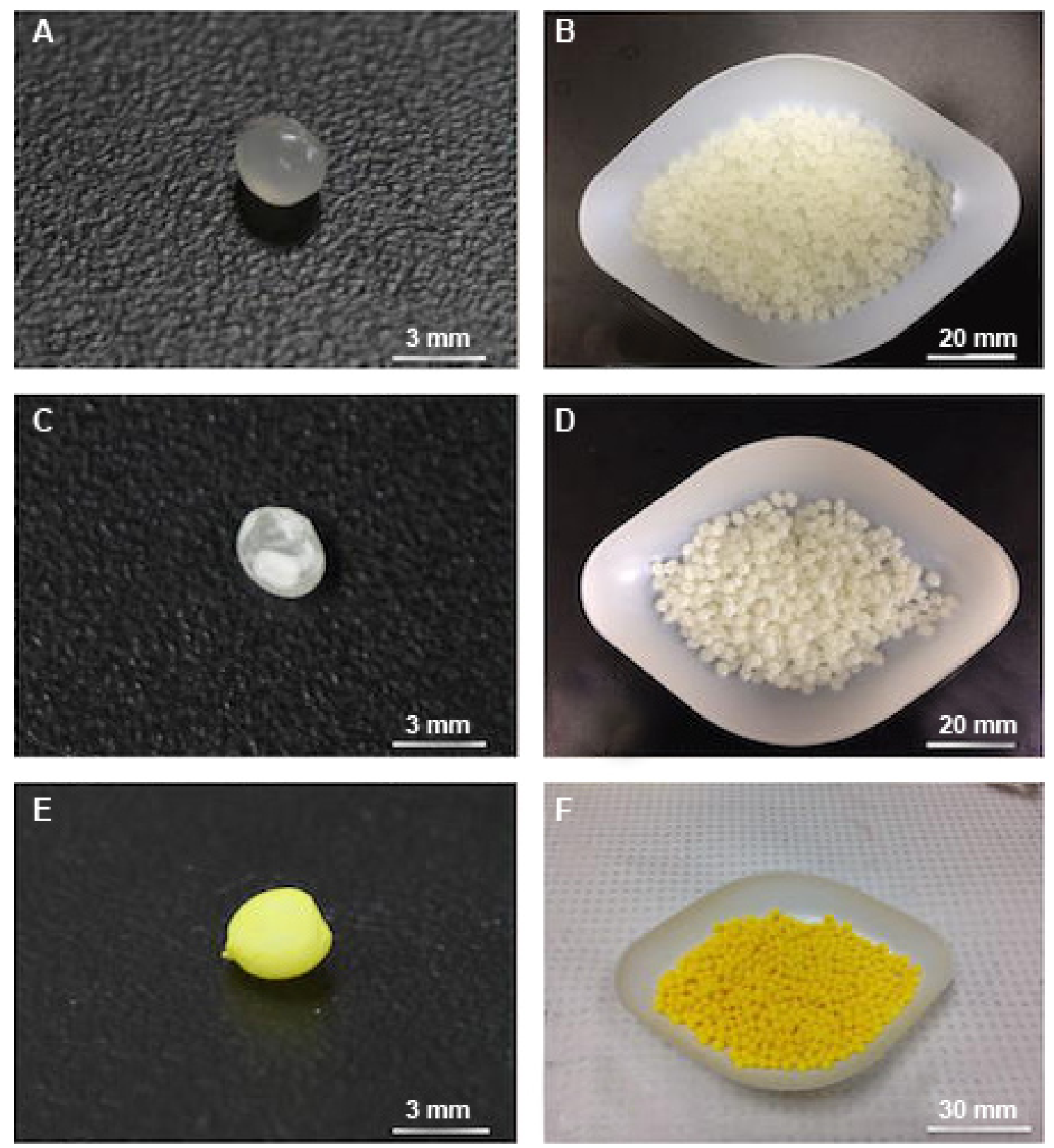

Figure 2 Photographs of polylactic acid pellets individually and in 20 gram batches.

Notes: (A, B) Control polylactic acid pellets. (C, D) 2.5 wt\% gentamicin-coated pellets. (E, F) 2.5 wt $\%$ methotrexate-coated pellets.

added to the plates for testing. Zones of inhibition were measured for the fabricated antibiotic test groups after 24 hours of incubation at $37^{\circ} \mathrm{C}$. Each sample group was tested in five plates for reliability and reproducibility.

\section{Liquid broth cultures}

Mueller Hinton culture broths were inoculated with $50 \mu \mathrm{L}$ of bacterial cultures and specimens of each group were added. These cultures were incubated for 24 hours at $37^{\circ} \mathrm{C}$ on a rocker. Triplicates of each group were tested and compared with controls.

\section{Osteosarcoma cell cultures}

An osteosarcoma cell line (CRL 2836) was purchased from the American Type Culture Collection (Manassas, VA, USA). Osteosarcoma cells were plated in $25 \mathrm{~cm}^{2}$ tissue culture flasks and incubated at $37^{\circ} \mathrm{C}$ under humidified $5 \% \mathrm{CO}_{2}$ and $95 \%$ air in complete Dulbecco's Modified Eagle's Medium containing 10\% fetal bovine serum and 1\% penicillin-streptomycin-amphotericin. Subconfluent cells were passaged with $0.25 \%$ Tryple-E, collected by centrifugation, resuspended in complete Dulbecco's Modified Eagle's Medium, and subcultured at a 3:1 split into $25 \mathrm{~cm}^{2}$ tissue 
culture flasks. Confluent cultures were passaged and seeded onto filaments at uniform cell density. Test wells were tested for toxicity using an XTT assay and the live/dead cytotoxicity assay on the third day of the experiment.

\section{XTT assay}

This assay is based on the ability of viable cells to reduce XTT dye. In these experiments, osteosarcoma cells were used. Cells were seeded in 96-well plates containing filaments with MTX along with other PLA filaments as controls. Cells were tested on day 3 when the wells were confluent. The XTT assay protocol supplied by the manufacturer (Sigma-Aldrich) was followed. After aspiration of medium, $100 \mu \mathrm{L}$ of buffer was added to each well along with $20 \mu \mathrm{L}$ of XTT solution. Photometric absorption of each well was measured at $450 \mathrm{~nm}$ and $690 \mathrm{~nm}$ after 4 hours of incubation. After completing the XTT assay, a live/dead cytotoxicity assay was conducted on additional wells of each category on the same plate.

\section{Live/dead cytotoxicity assay}

A live/dead cytotoxicity kit was used for testing. The protocol supplied by the manufacturer was used for the assay. The dyes were diluted to $2 \mu \mathrm{M}$ solutions and were added to the test wells. Fluorescence microscopy was used to image live and dead cells after they were incubated for 30 minutes in the dye solutions.

\section{Additive heating}

Additives were heated for thermal stability testing using a clay Vulcan oven that had been preset to a desired temperature. The GS was placed in the Vulcan oven for

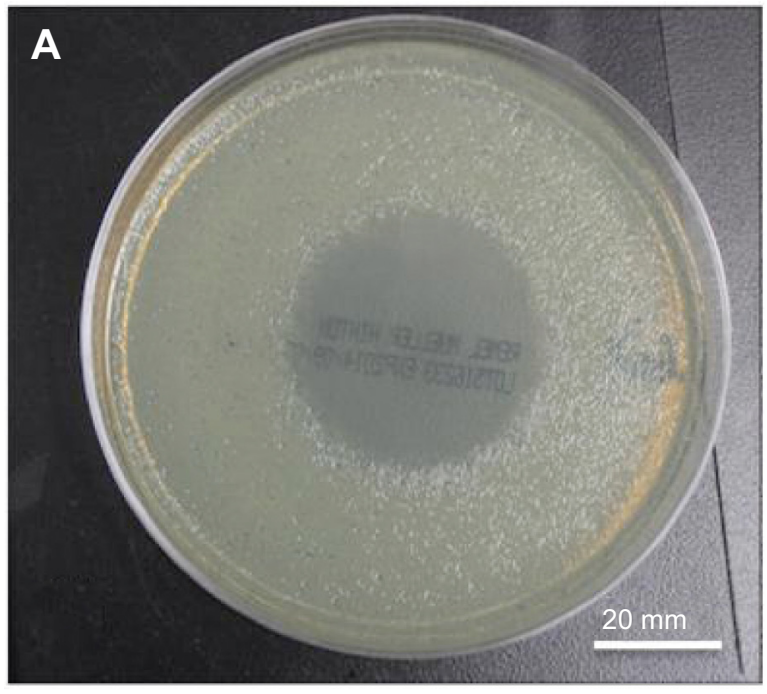

Figure 3 Bacterial plates.

Notes: (A) Control I mg gentamicin powder. (B) Heated I mg gentamicin powder.
5 minutes after it had been heated to $220^{\circ} \mathrm{C}$. The MTX was placed in the Vulcan oven for 5 minutes after it had been heated to $190^{\circ} \mathrm{C}$.

\section{Statistical analysis}

Multiple values were taken for necessary experiments and averages were compared using one-way analysis of variance at $\alpha=0.05$. The statistical analysis was performed using Minitab $^{\circledR}$ software.

\section{Results \\ Quantification of thermal stability of antibiotics}

GS antibiotics were tested for short-term thermal stability by heating for 5 minutes in a Vulcan clay oven preheated to $220^{\circ} \mathrm{C}$. The GS was ground with a mortar and pestle and $100 \mathrm{mg}$ of the antibiotic was heated in a sterile glass beaker. Next, $1 \mathrm{mg}$ samples were weighed out and added into Mueller-Hinton plates or test tubes containing Mueller Hinton broth. The plates or broths had already been inoculated with E. coli according to ISO standard 17025. Control unheated GS was also added to a separate set of broths or plates. After incubating overnight at $37^{\circ} \mathrm{C}$, the cultures were checked for growth. No growth occurred in any of the broth cultures, and all plates had strong zones of inhibition. The diameter of the zone of inhibition on each sample was measured three times and averaged $(n=3)$. The control GS had an average zone of inhibition of $37.39 \pm 0.55 \mathrm{~mm}$ while the heated GS had a zone of inhibition of $36.61 \pm 1.56 \mathrm{~mm}$, as shown in Figure 3. Analysis of variance was performed and showed no difference between the two groups. Additional

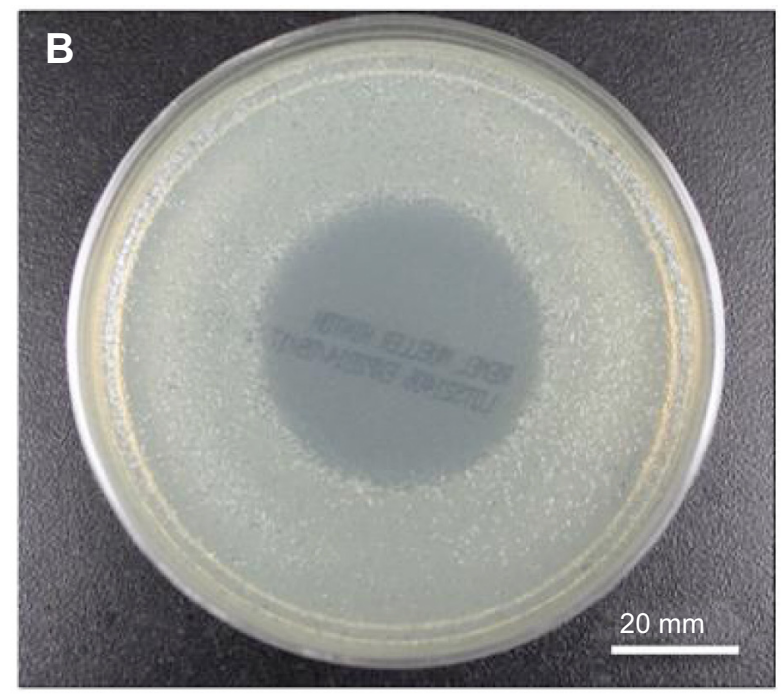


control plates with bacteria, no bacteria, and $100 \mu \mathrm{g}$ control filter paper GS-eluting discs were prepared to verify results. The zone of inhibition was $29.83 \pm 0.34 \mathrm{~mm}$ for the control filter paper-eluting GS discs.

\section{Testing of pellet coating with antibiotics}

The extrusion process required consistent coating of the PLA pellets with additives. We used silicone-coating oil for coating of the desired additive. Tests were performed to check the PLA pellets and silicone coating for antibacterial properties. Tests were also performed to check the consistency of the GS coating on the PLA pellets. SEM was used to document that coatings were applied to the pellets as shown in Figure 4 and that they appeared consistent. It should be noted that before SEM, the samples needed to be cleaned with compressed air which removed some of the powdered coating. Figure 4C clearly shows a consistent coating of gentamicin powder on a pellet.

Plain PLA pellets, PLA pellets coated in silicone oil, and PLA pellets coated in GS additive were added to Mueller
Hinton plates or broth cultures. The results showed no zone of inhibition in plates or broth cultures with PLA pellets or PLA pellets coated in silicone oil (Figure 5). The pellets coated in $2.5 \mathrm{wt} \%$ GS showed no growth in the broth culture and a strong zone of inhibition averaging $29.03 \pm 2.78 \mathrm{~mm}$ on the plates, as shown in Figure 6. Analysis of variance showed a significant difference between antibiotic coating and all other groups as well as a consistent coating based on zones of inhibition.

\section{Filament extrusions and testing}

The plain PLA pellets, silicone oil only-coated PLA pellets, and GS-coated PLA pellets were extruded at $175^{\circ} \mathrm{C}$ through the filament extruder sequentially to prevent additive contamination of the controls. The extrusion occurred in a sterile fume hood, and the resulting filaments were put into sterile plastic containers. Filaments were cut into $10 \mathrm{~mm}$ lengths weighing on average $50 \mathrm{mg}$, and SEM was then done as shown in Figure 7. Mueller
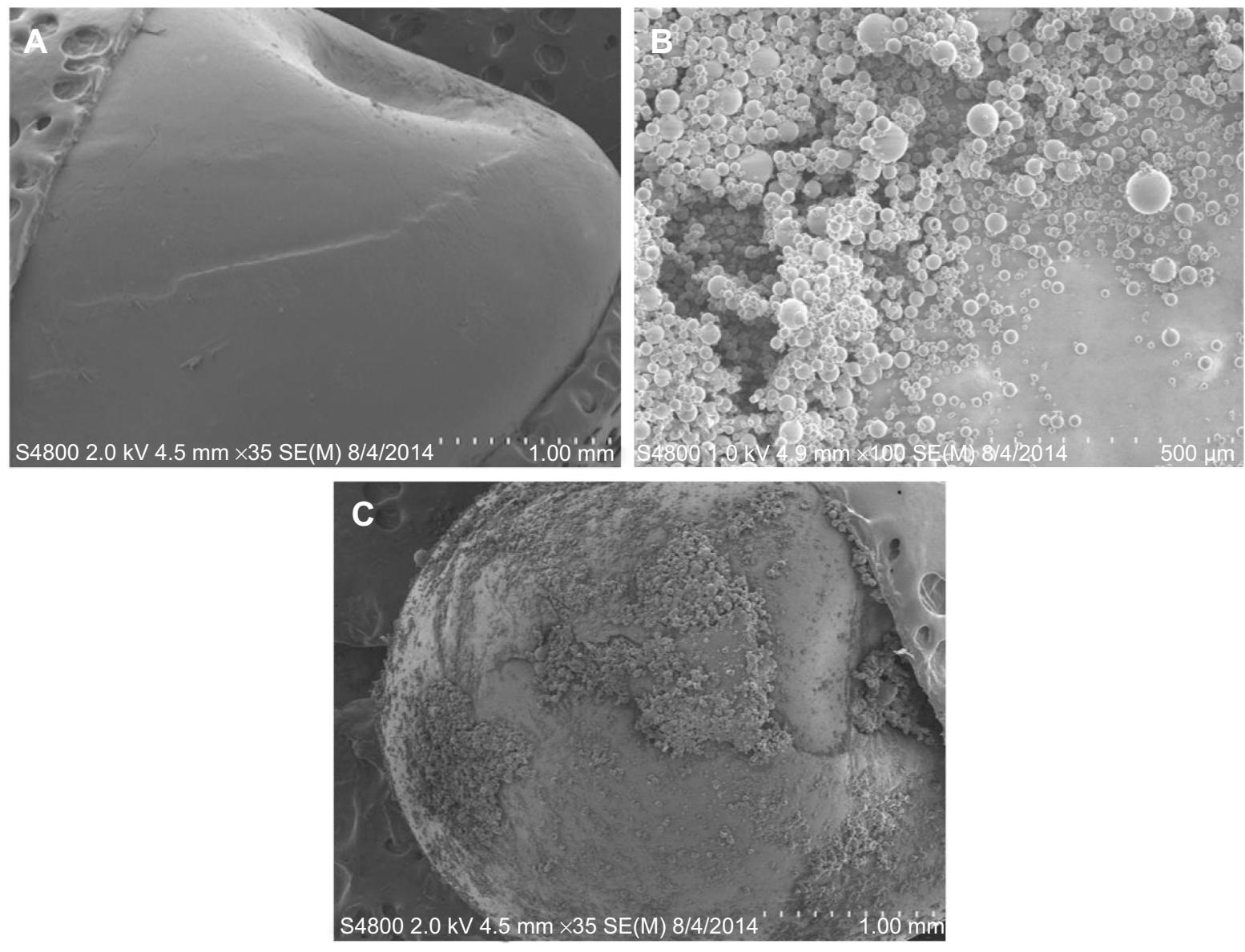

Figure 4 Scanning electron micrographs of pellets and coated pellets.

Notes: (A) Control polylactic acid pellet. (B) Gentamicin powder. (C) 2.5 wt\% gentamicin-coated pellet. 

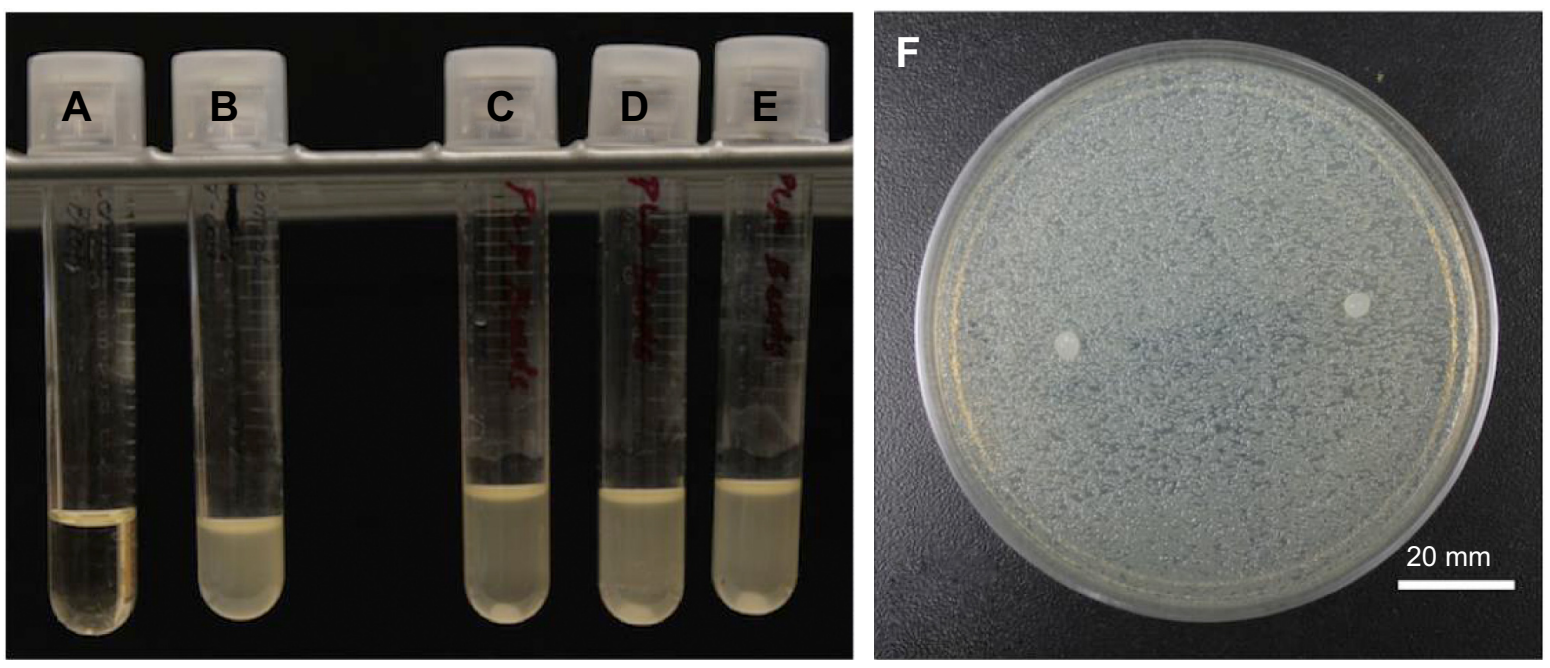

Figure 5 Bacterial cultures and bacterial cultures with control and oil-coated pellets.

Notes: (A) Control broth. (B) Bacteria-inoculated broth. (C-E) Bacteria-inoculated broth with control PLA pellets. (F) PLA control pellet on left and a PLA oil-coated pellet on right.

Abbreviation: PLA, polylactic acid.

Hinton plates and broth cultures were prepared according to ISO standard 17025 and inoculated with E. coli, as were control plates. Five plates or broth cultures were used for each filament type. The plain PLA filament and silicone oil containing PLA filament showed no bacterial inhibition. The $2.5 \mathrm{wt} \%$ GS containing filament had no growth in broth cultures and a zone of inhibition on bacterial plates that averaged $23.12 \pm 0.79 \mathrm{~mm}$ based on three diameter measurement points. These results are shown in Figures 8 and 9. Analysis of variance showed a statistically significant difference between the PLA, silicone-PLA, and GS-PLA filaments, as well as no difference in zone of inhibition size among the PLA and silicone-PLA filaments.

\section{D fabrications of discs and bacterial testing}

Fabricated PLA filaments were found to be within a diameter of $1.75 \pm 0.1 \mathrm{~mm}$. This was detected by measuring each filament with digital calipers every few centimeters and
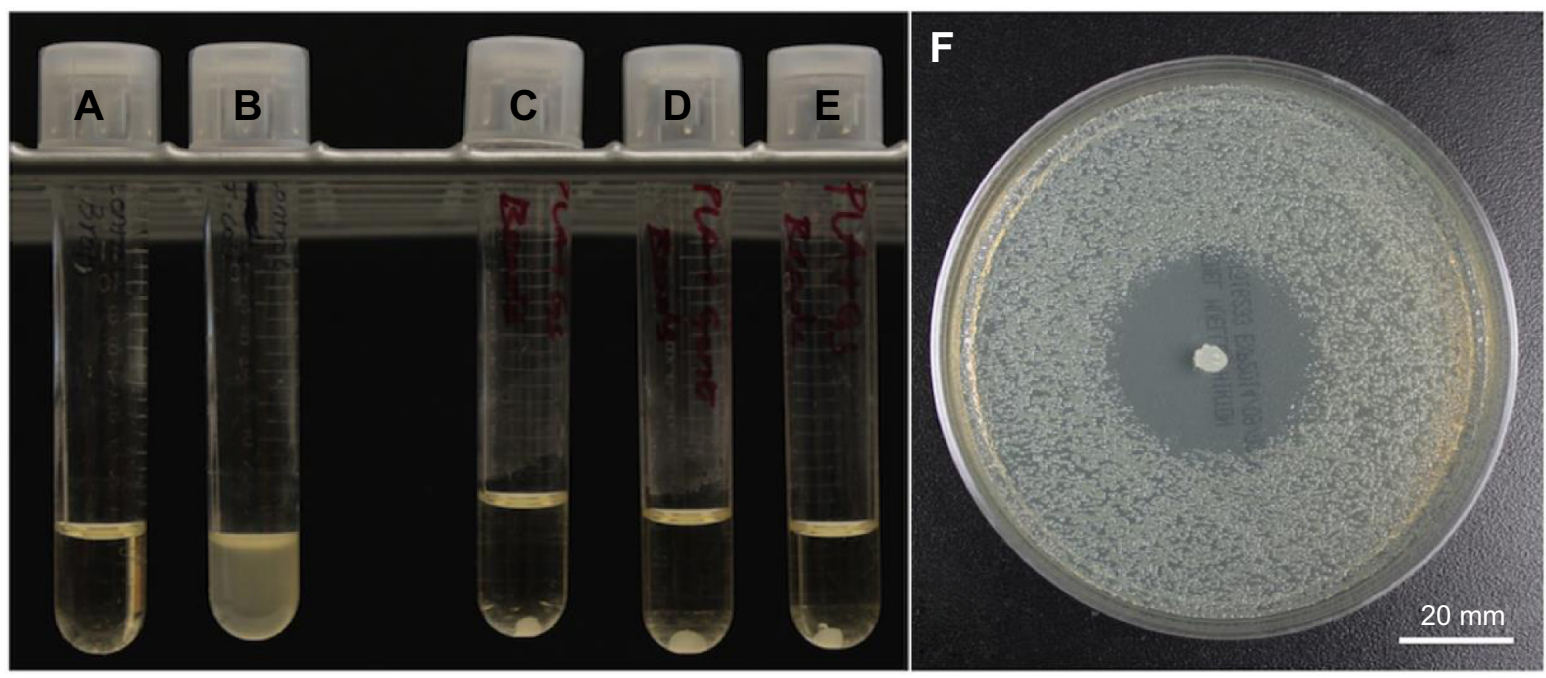

Figure 6 Bacterial broth cultures and bacterial cultures with control and gentamicin-coated pellets.

Notes: (A) Control broth. (B) Bacteria-inoculated broth. (C-E) Bacteria-inoculated broth with 2.5 wt\% gentamicin-coated PLA pellets. (F) 2.5 wt\% gentamicin-coated PLA pellet.

Abbreviation: PLA, polylactic acid. 

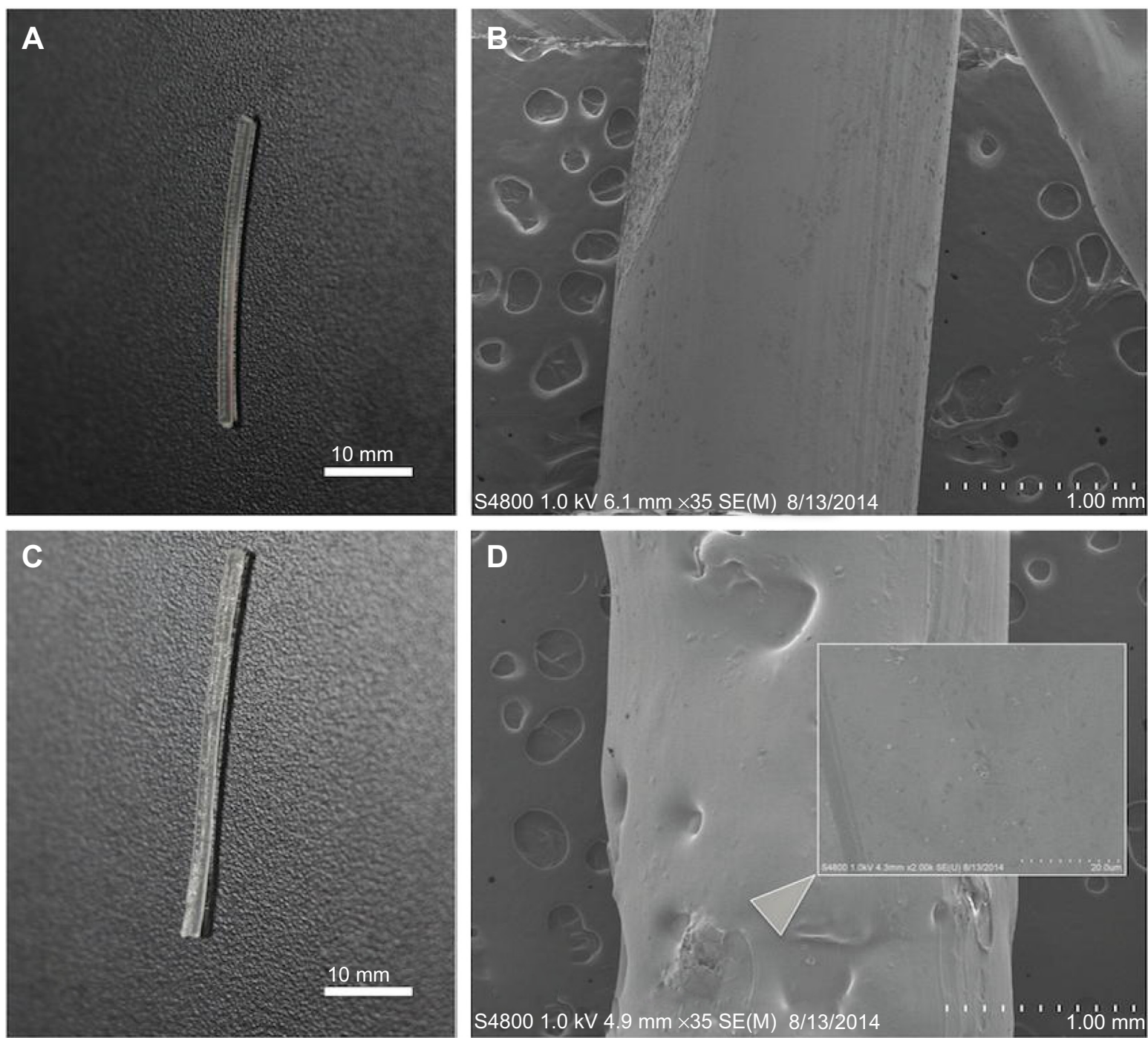

Figure 7 Photographs and SEM of control PLA filaments and gentamicin-laden filaments.

Notes: (A) Photograph of control PLA filament. (B) SEM of control PLA filament. (C) Photograph of 2.5 wt\% gentamicin-laden filament. (D) SEM 2.5 wt\% gentamicin-laden filament showing gentamicin embedded in the filament.

Abbreviations: PLA, polylactic acid; SEM, scanning electron micrograph.

confirmed by the fact that loading into the feed mechanism of the 3D printer would not work with filaments that varied significantly in diameter. The filaments were test-loaded into a Makerbot $2 \mathrm{X}$ fused deposition-modeling printer. All filaments were able to test-load with little to no clogging. SolidWorks was used to design a $5 \mathrm{~mm}$ diameter, $1 \mathrm{~mm}$ height test disc. The disc file was printed using the control factory filament with the printing specifications being a $220^{\circ} \mathrm{C}$ printer extrusion head temperature, 300-micron layer height, and the $100 \%$ fill setting. No rafts or supports were used, and the printer bed was sterilized with alcohol prior to filament loading and printing. The design and printing process is shown in Figure 10. The plain PLA and PLA with GS additive filaments were then printed into $5 \mathrm{~mm}$ discs. Discs were placed into sterile $1 \mathrm{~mL}$ Eppendorf tubes for storage. Mueller Hinton plates and broth cultures were prepared to ISO standard 17025 and inoculated with E. coli, as were control plates. Five plates or broth cultures were used for each disc type. The plain PLA $5 \mathrm{~mm}$ discs showed no bacterial inhibition in any of the broth cultures on agar plates. The discs made from 1 $\mathrm{wt} \%$ and $2.5 \mathrm{wt} \%$ GS containing filament had no growth in broth cultures and a zone of inhibition on bacterial plates that averaged $12.9 \pm 2.56 \mathrm{~mm}$ and $21.35 \pm 1.0 \mathrm{~mm}$, respectively, based on three separate diameter measurements (Figures 11 and 12). Analysis of variance showed a statistically significant difference between the PLA and GS-PLA discs. 


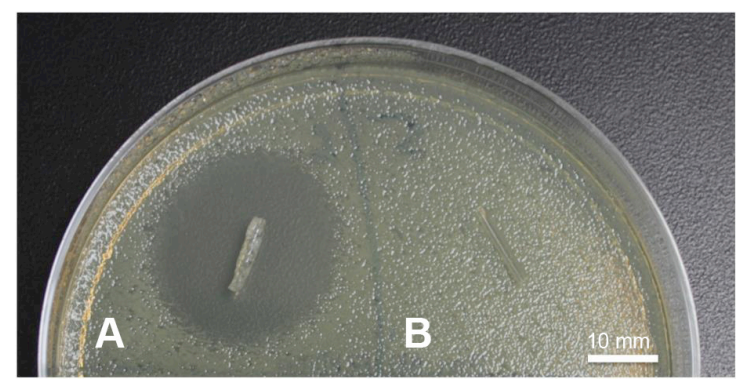

Figure 8 Bacterial culture containing a control PLA and a gentamicin-laden PLA filament.

Notes: (A) 2.5 wt\% gentamicin-laden PLA filament. (B) Control PLA filament. Abbreviation: PLA, polylactic acid.

\section{D fabrications of antibacterial catheters and antibiotic beads}

For the $5 \mathrm{~mm}$ discs, typical print time was in the order of about one minute per construct. To test more complicated printing with this filament, other medically relevant constructs were fabricated using a $1 \mathrm{wt} \%$ GS filament. SolidWorks was again used to fabricate a $6 \mathrm{~mm}$ spherical antibiotic bead with a $3 \mathrm{~mm}$ lumen. A 14 French partial surgical catheter was also designed with a single drainage hole near the distal tip with a length of $20 \mathrm{~mm}$. The 3D printer was set for fabrication at a $300-$ micron resolution and a $10 \%$ in-fill software setting for these objects. Several antibiotic beads and surgical catheters were constructed from both a factory PLA filament and a GS-PLA filament. The SolidWorks renderings and their associated printed constructs are shown in Figure 13.

\section{Thermal stability of MTX}

MTX powder was heated in a Vulcan oven to $190^{\circ} \mathrm{C}$ for 5 minutes. The heated MTX along with a control MTX

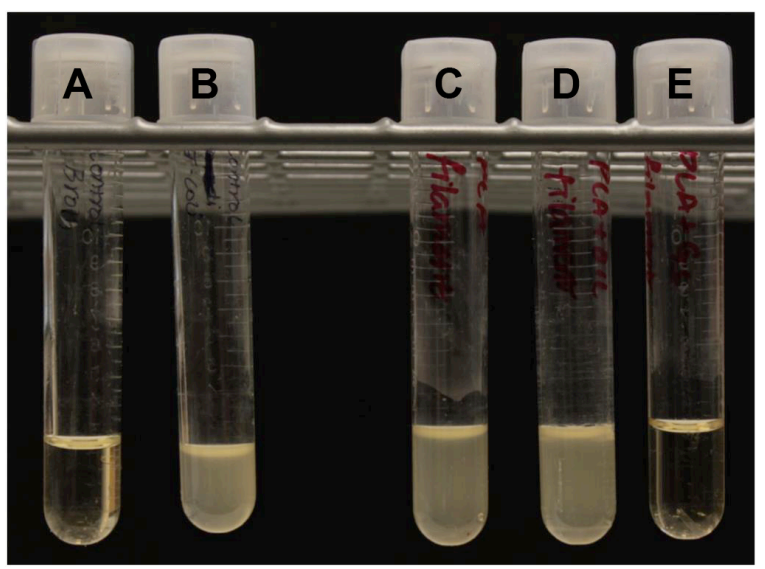

Figure 9 Bacterial broth cultures containing control, oil-coated, and gentamicinladen PLA filaments.

Notes: (A) Control broth. (B) Bacteria-inoculated broth. (C) Control PLA filament. (D) Oil-coated PLA filament. (E) 2.5 wt\% gentamicin-doped PLA.

Abbreviation: PLA, polylactic acid.
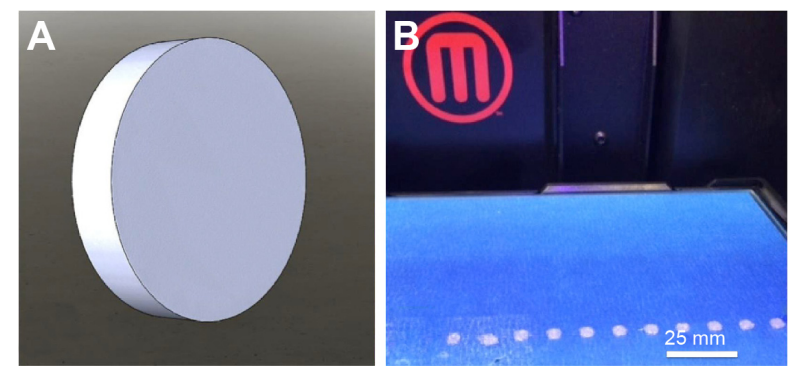

Figure 10 Design disc and resultant three-dimensional printed discs. Notes: (A) Disc design file. (B) Printed discs on printer platform.

powder was added to osteosarcoma cells that had been plated in a 96-well plate. After a day, an XTT was completed to check cell activity. Inhibition of osteosarcoma growth by both heated and control MTX was found (Figure 14). Compared with controls, inhibition of activity by heated and control MTX was $65.41 \%$ and $61.17 \%$, respectively. Analysis of variance was run to confirm that there was both statistically significant inhibition of growth and no statistically significant difference between groups. A live/dead assay was run to demonstrate any differences between the control, MTX control, and MTX heated groups (Figure 15). There was a nominal difference between the two MTX groups.

\section{MTX filament extrusion}

A filament required for 3D printing of PLA was doped with a $2.5 \%$ MTX content. PLA pellets were coated with $20 \mu \mathrm{L}$ of silicone oil and mixed with $500 \mathrm{mg}$ of MTX. These coated pellets were extruded with the ExtrusionBot to obtain a filament with a diameter of $1.75 \mathrm{~mm}$ at $160^{\circ} \mathrm{C}$. This filament was kept sterile for future plating. A different ExtrusionBot filament extruder was used to ensure no cross contamination with the GS-manufactured filaments. Sterile control filaments of plain extruded PLA and PLA pellets with silicone oil were taken from the extrusions done for the GS experiments and

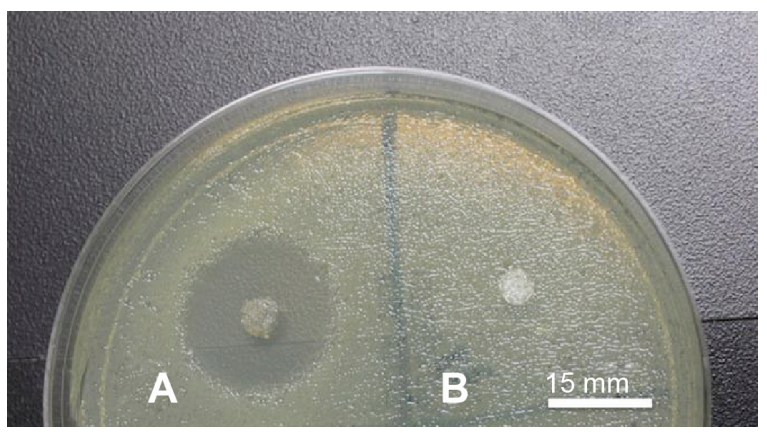

Figure I I Gentamicin-laden and control PLA discs.

Notes: (A) 2.5 wt\% gentamicin-laden PLA disc. (B) Control PLA disc. Abbreviation: PLA, polylactic acid. 


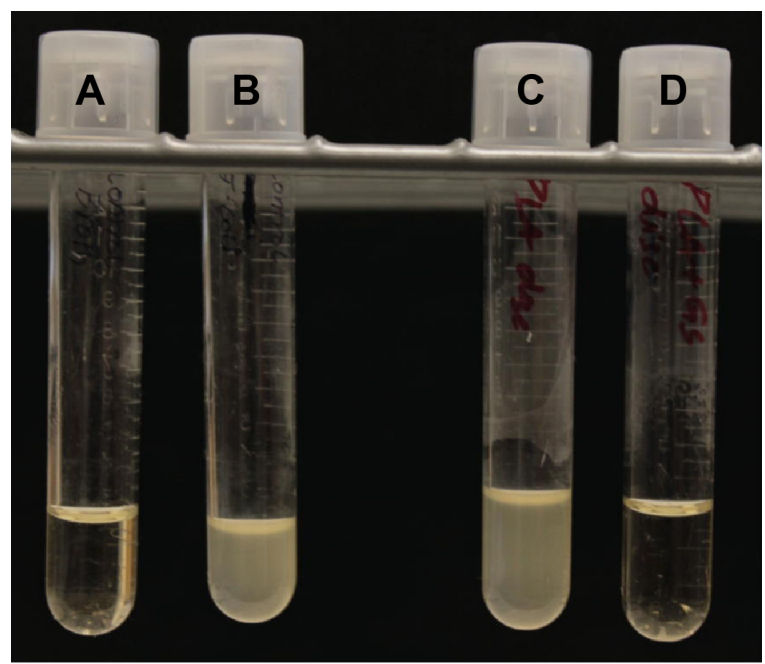

Figure 12 Control and gentamicin laden discs in bacterial growth culture. Notes: (A) Control broth. (B) Bacteria-inoculated broth. (C) Control PLA filament disc. (D) 2.5 wt\% gentamicin-laden PLA disc.

Abbreviation: PLA, polylactic acid.

placed aside for MTX cell studies. Pictures and SEM imaging of the filament was done as shown in Figure 16. The MTX crystals can be seen upon SEM of the filament.

\section{Osteosarcoma cell studies with MTX-PLA filaments}

MTX-enhanced PLA filaments were tested with osteosarcoma cells plated on 96-well plates. For this portion of the study, we used eight test groups with five wells tested: control cells, control MTX, heated MTX, control PLA pellet, oil-coated PLA pellet, MTX-coated PLA pellet, control PLA filament, and MTX-PLA filament. After 3 days, once the control osteosarcoma well was confluent, an XTT and live/dead assay was done. The XTT assay showed enhanced osteosarcoma growth in the wells not containing MTX (Figure 17). Silicon oil showed no effect on cell growth, and this was shown by comparing a control PLA pellet with a pellet coated in silicon oil. The control PLA filament showed no difference in cell proliferation compared with the control well. There was a significant decrease in cell proliferation in the well with MTX-enhanced PLA filament when compared with both the control well and the PLA filament control well. There was a significant decrease in cell proliferation in the well with MTX-enhanced PLA filaments when compared with both the control well (no filament) and the control well containing only PLA filaments.

\section{D fabrication of chemotherapeutic beads} As proof of principle, an MRX-laden bead was fabricated. SolidWorks was used to fabricate a $6 \mathrm{~mm}$ spherical antibiotic bead with a $3 \mathrm{~mm}$ lumen. That bead was printed on a Makerbot 2X 3D printer set to $190^{\circ} \mathrm{C}$ with external pressure on the filament, and is shown next to a gentamicin-laden bead in Figure 18.
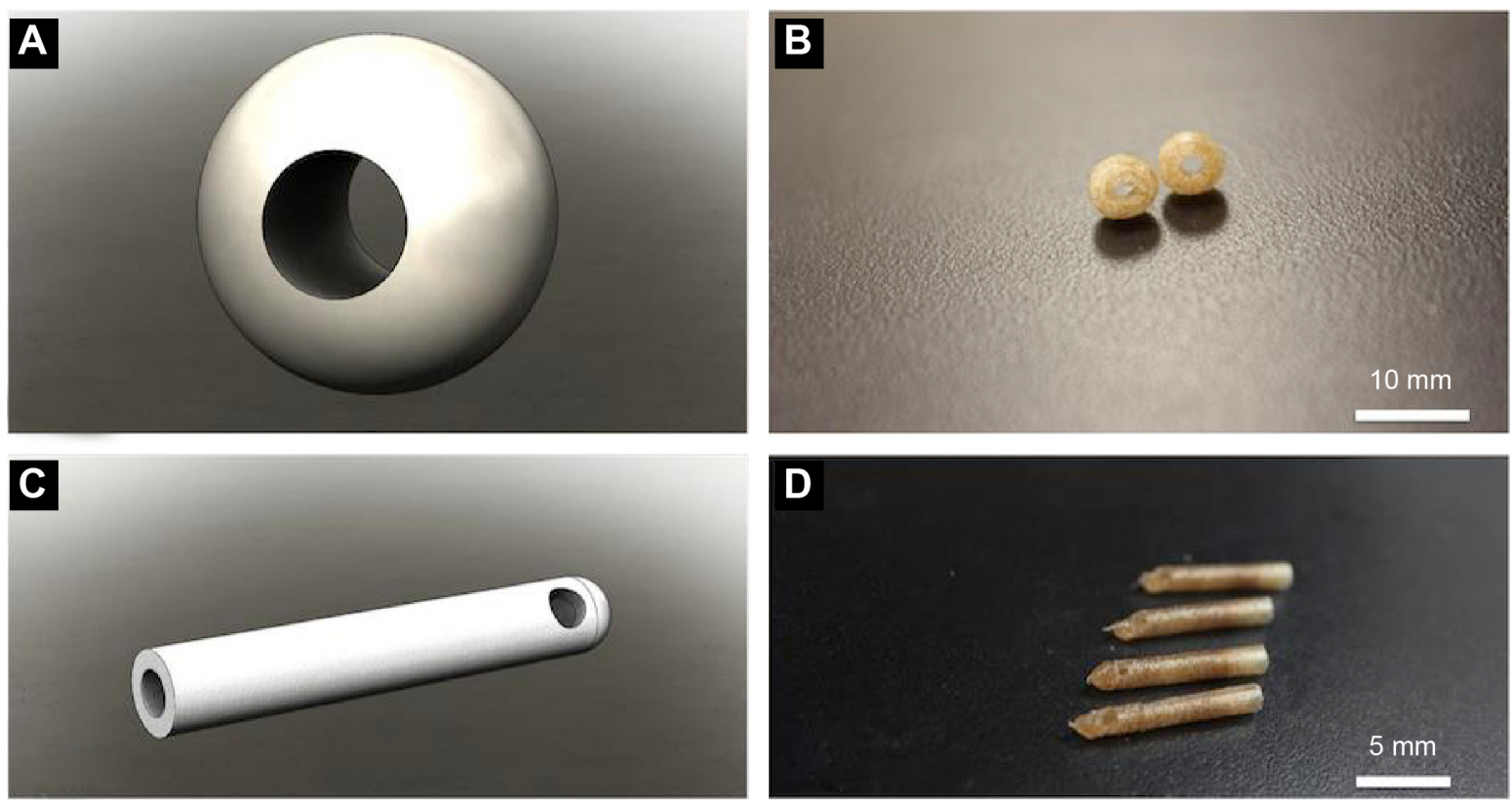

Figure 13 Design files and resultant gentamicin-laden PLA filament and catheters.

Notes: (A) Bead design file. (B) Printed I wt\% gentamicin-laden PLA beads. (C) Catheter design file. (D) Printed I wt\% gentamicin-laden PLA catheters. Abbreviation: PLA, polylactic acid. 


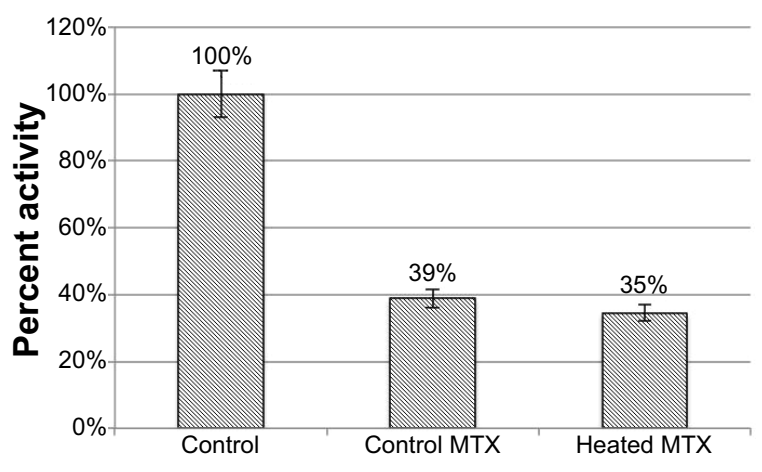

Figure I 4 XTT table showing percent activity.

Abbreviation: MTX, methotrexate.

\section{Discussion}

Our objective was to develop an antibiotic-eluting and chemotherapeutic-eluting filament that would maintain integrity throughout the extrusion and $3 \mathrm{D}$ printing process. The ultimate purpose of these drug-loaded filaments is to enable ease of fabrication for patient-specific biocompatible treatment constructs for personalized medicine. The first step in reaching this goal was developing a filament extrusion process that results in consistent dispersion of the additive without thermal degradation of the additive, while also maintaining a $1.75 \mathrm{~mm}$ diameter. This was achieved by use of commercially available PLA pellets coated in a silicone oil, and suspension of the additives was achieved on the surface of the pellets in such a way that, once vortexed, provides an even dispersion throughout the batch. As discussed previously, extrusion temperatures were a key consideration so as to ensure minimal degradation of GS and MTX.

Since the typical extrusion temperature of PLA for 3D printing filaments is $175^{\circ} \mathrm{C}$, the process of using lower temperatures resulted in a slightly slower extrusion speed of the filaments. Incorporation of the oil coating showed that addition of too much oil, typically $>20 \mu \mathrm{L}$, compromised the fidelity of the filament as the oil flowed more readily than the plastic pellets. This resulted in a caramelized substance that flowed freely through the die of the extruder, taking the majority of the additive with it. To prevent this, coatings were limited to $<20 \mu \mathrm{L}$.

Introduction of a loaded filament or printed disc to the respective bacterial cell culture resulted in well-defined zones of inhibition, confirming that the 3D-printed constructs retained the antibacterial properties of the additives in such concentrations as to prove medically viable. When comparing the filaments with the printed structures tested in the same environments, the printed structures proved to have comparable zones of inhibition, supporting the conclusion that the printing process does not further degrade additive effectiveness. To ensure that nothing in the manufacturing process was extraneously influencing the zone of inhibition, a batch of pellets coated solely in silicone oil were extruded, and the powdered forms of the additives were tested in the same culture environment after having been placed in an oven preheated to $220^{\circ} \mathrm{C}$, far above the extrusion temperature, and on par with the printing temperature.

While the tested doping percentages were relatively low ( $1 \%$ and $2.5 \%$ by weight), the effectiveness of inhibition was found to be relatively high. Should greater doping percentages be desired, a modified coating method can be used to layer stack oil and an additive atop the desired plastic pellet. It should be noted that toxicity is a common issue with systemic drug delivery, but by constraining the dopant percentage, this should allow for a higher dosage to a desired area than a systemic treatment. 3D-printed constructs could be fabricated with various percentage fills and at a variety of resolutions. Consumer 3D printers have resolutions ranging from 50 to 400 microns with percentage fill setting options ranging from $10 \%$ to $100 \%$. Commercial printers have higher resolutions and could allow for more customization. Different fill percentages or differing geometries may alter drug elution
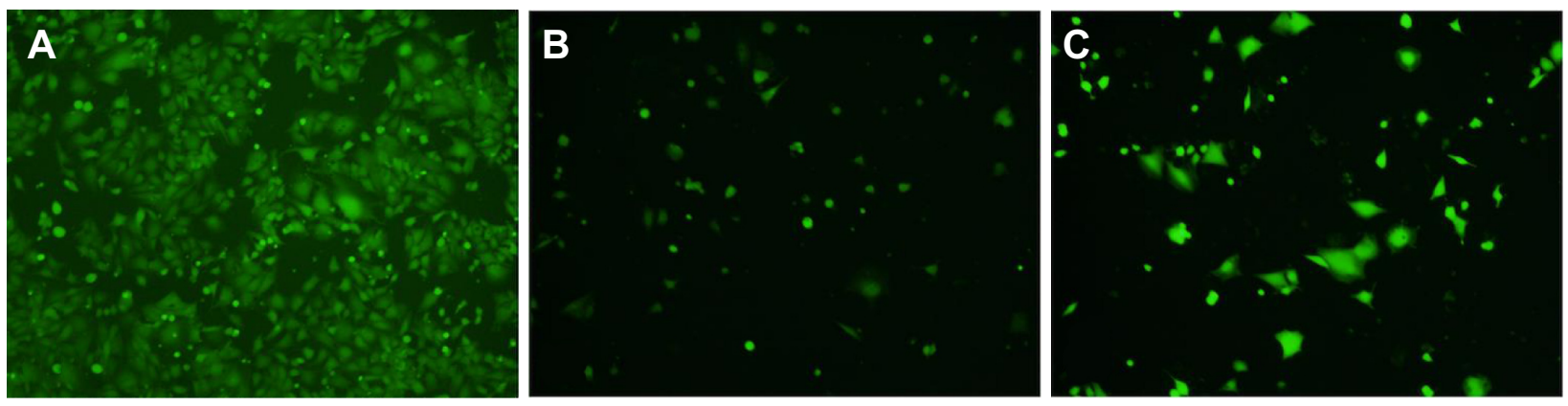

Figure 15 Live/dead cytotoxicity assays.

Notes: (A) Control well. (B) Control methotrexate. (C) Heated methotrexate. 

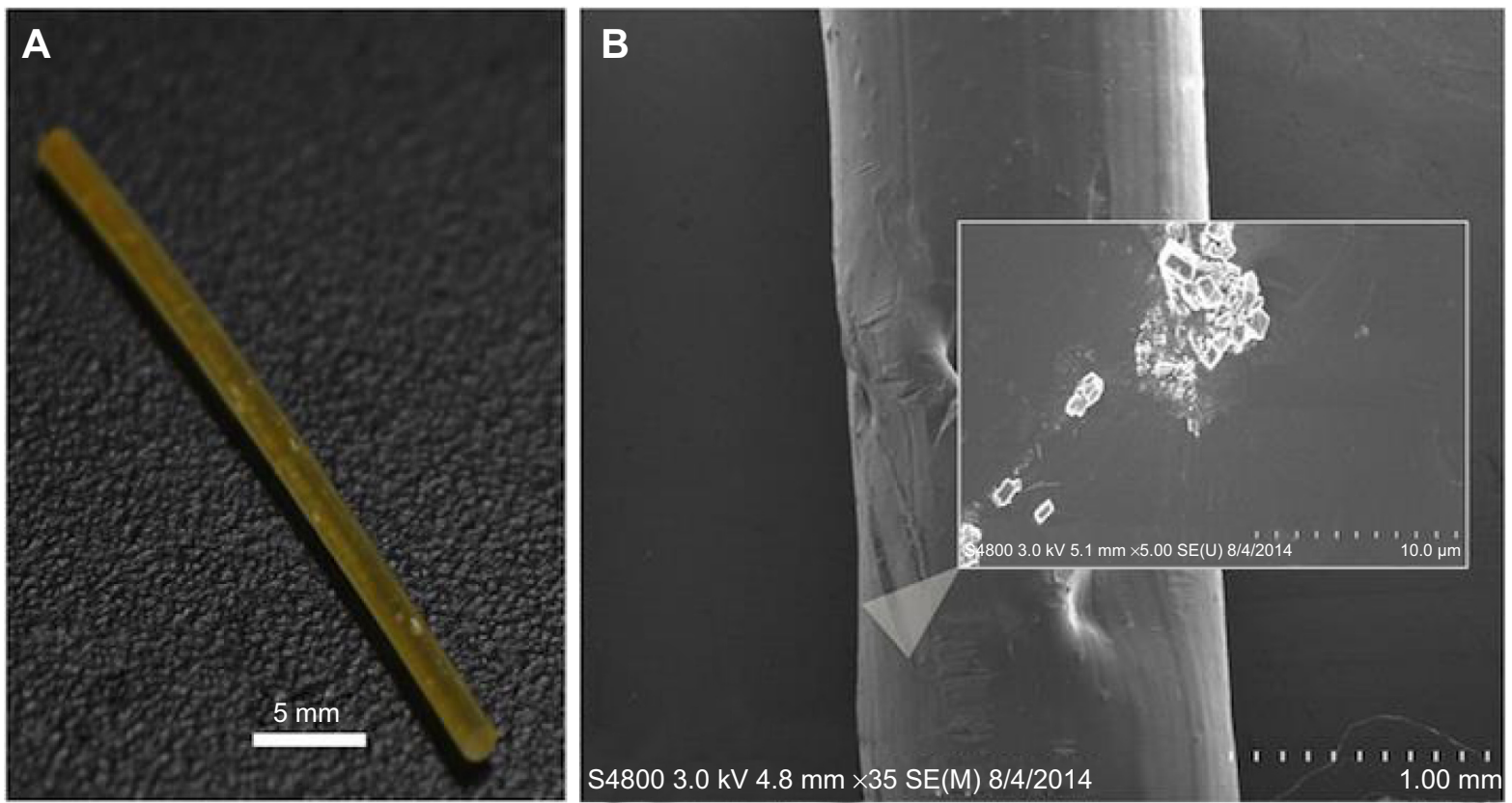

Figure 16 Photograph and SEM of 2.5 wt\% methotrexate PLA filament.

Notes: (A) 2.5 wt\% methotrexate PLA filament. (B) SEM of 2.5 wt\% methotrexate PLA filament showing embedded methotrexate.

Abbreviations: PLA, polylactic acid; SEM, scanning electron micrograph.

rates. Fabrication of medical implants with different percent fills would allow for a greater surface area for potential bioactive compound elution. The tunable nature of this type of fabrication can allow for custom construct fabrication that would not be possible in mass production.

Future studies of this process looking at specific elution profiles of various additive-loaded filaments must be performed to ensure proper characterization of the release in targeted treatments based upon the fabrication process. Validation in an in vivo osteomyelitis study is also required.
MTX-laden filaments were also created to show the potential for customized fabrication of chemotherapeuticeluting constructs. The XTT and live/dead kits showed comparable activity from MTX powders that had been heated similarly during the fabrication process. The MTX-laden filaments prevented growth, as was shown by analysis of variance when compared with controls. MTX is primarily cytostatic as opposed to cytotoxic. Measurements were taken once the control wells had reached near confluence. Future work with cytotoxic compounds may show more

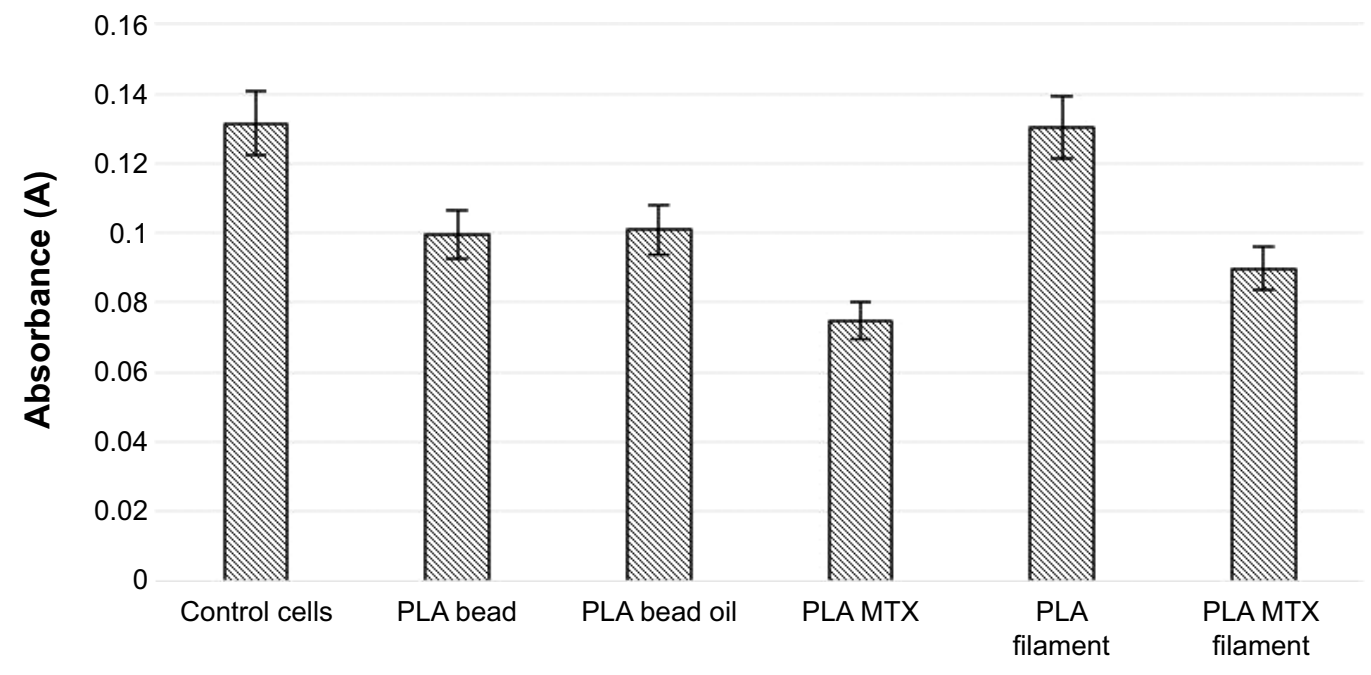

Figure 17 XTT assay test.

Abbreviations: PLA, polylactic acid; MTX, methotrexate. 


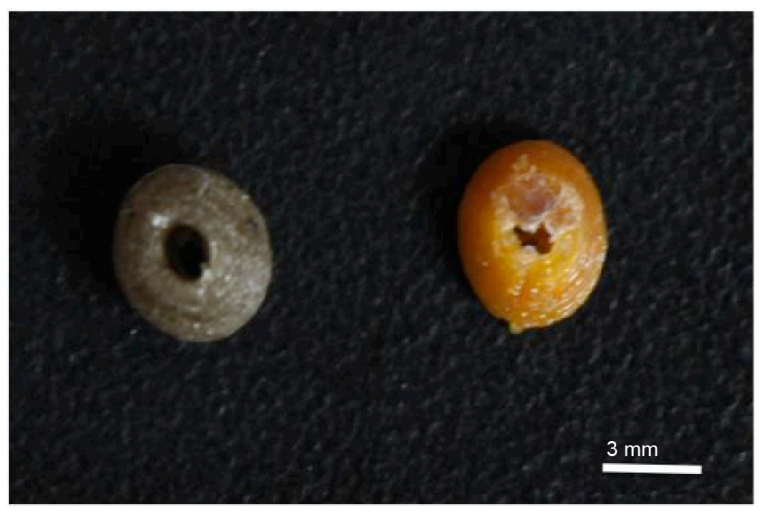

Figure 18 Comparison between gentamicin-laden and methotrexate-laden beads. Left image represents a gentamicin-laden bead and the right image represents a methotrexate-laden bead.

significant reductions in cell activity on both the live/dead and XTT assays. Additionally fabrication of test discs with a number of layers or hollow constructs would likely increase the surface area, increase elution, and result in a decrease in cell activity.

\section{Conclusion}

3D printing of antibiotic filaments showed zones of inhibition on par with direct introduction of powdered forms of the additives into the cultured environment. 3D filament extrusion of PLA-MTX filaments also showed inhibition of cancer cell growth. Testing of the oil used to coat the PLA pellets showed that the effects are entirely additive-based. Applications of this fabrication process may enable fabrication of patient-specific treatment implants to target infection. By using PLA, a wellknown biocompatible plastic, as the primary component of the implants, normal body functions should be able to break down the implant after time, negating the necessity of additional procedures to remove the implant. An understanding of the elution profiles of additives is necessary in order to fully comprehend patient treatment via this method.

\section{Acknowledgments}

The authors would like to thank Jeffery Ambrose, Lin Sun, and Mark DeCoster of Louisiana Tech University for their assistance with the cell culturing techniques.

\section{Author contribution}

All authors contributed to the research, data analysis, and writing of this study.

\section{Disclosure}

The authors report no conflicts of interest in this work.

\section{References}

1. Sachs EM, Haggerty JS, Cima MJ, Williams PA. Three-dimensional printing techniques. US Patent No 5,340,656, 1994.

2. Jijotiya D, Verma PL. A survey of performance based advanced rapid prototyping techniques. Sch J Eng Tech. 2013;1:4-12.

3. Dimitrov D, Schreve K, DeBeer N. Advances in three-dimensional printing-state of the art and future perspectives. Rapid Prototyping J. 2006;12:136-147.

4. Kitson PJ, MacDonnell A, Tsuda S, Zang HY, Long DL, Cronin L. Bringing crystal structures to reality by three-dimensional printing. Cryst Growth Des. 2014;14:2720-2725.

5. Zhang AP, Qu X, Soman P, et al. Rapid fabrication of complex 3D extracellular microenvironments by dynamic optical projection stereolithography. Adv Mater. 2012;24:4266-4270.

6. Dias AD, Kingsley DM, Corr DT. Recent advances in bioprinting and applications for biosensing. Biosensors. 2014;4:111-136.

7. Ozbolat IT, Yu Y. Bioprinting towards organ fabrication: challenges and future trends. IEEE Trans Biomed Eng. 2013;60:691-699.

8. Selimovic S, Saedinia S, Mehmet S, Dokmeci R, Khademhosseini A. Microfabricated microvasculature networks. Lab Chip. 2012;12: 3217-3220.

9. Derby B. Printing and prototyping of tissues and scaffolds. Science. 2012;338:921-925.

10. Bose S, Vahabzadeh S, Bandyopadhyay A. Bone tissue engineering using 3D printing. Mater Today (Kidlington). 2013;16:497-504.

11. Landers R, Mülhaupt R. Desktop manufacturing of complex objects, prototypes and biomedical scaffolds by means of computer-assisted design combined with computer-guided 3D plotting of polymers and reactive oligomers. Macromol Mater Eng. 2000;282:17-21.

12. Ahn BY, Duoss EB, Motala MJ, et al. Omnidirectional printing of flexible, stretchable, and spanning silver microelectrodes. Science. 2009;323:1590-1593.

13. Hayhurst C. 3D printing and medical devices. Biomed Instrum Technol. 2014;48:14-24.

14. Marais LC, Ferreira N, Aldous C, Le Roux TLB. The pathophysiology of chronic osteomyelitis. SA Orthop J. 2013;12:14-18.

15. Gomes D, Pereira M, Bettencourt AF. Osteomyelitis: an overview of antimicrobial therapy. Braz J Pharm Sci. 2013;49:13-27.

16. Garvin K, Feschuk C. Polylactide-polyglycolide antibiotic implants. Clin Orthop. 2005;437:105-110.

17. Adams K, Couch L, Cierny G, Calhoun J, Mader JT. In vitro and in vivo evaluation of antibiotic diffusion from antibioticimpregnated polymethylmethacrylate beads. Clin Orthop. 1992;278: 244-252.

18. Tsourvakas S. Local Antibiotic Therapy in the Treatment of Bone and Soft Tissue Infections, Selected Topics in Plastic Reconstructive Surgery, Dr Stefan Danilla (Ed.) 2012; InTech Publishers.

19. Tsourvakas S. Local antibiotic therapy in the treatment of bone and soft tissue infection. In: Danilla S, editor. Selected Topics in Plastic Reconstructive Surgery. Intechopen Publishers; 2000. Available from: http:// www.intechopen.com/books/selected-topics-in-plastic-reconstructivesurgery/local-antibiotic-therapy-in-the-treatment-of-bone-and-softtissue-infections. Accessed November 30, 2014.

20. Blaha JD, Calhoun JH, Nelson CL, et al. Comparison of the clinical efficacy and tolerance of gentamicin PMMA beads on surgical wire versus combined and systemic therapy for osteomyelitis. Clin Orthop. 1993;295:8-12.

21. Mader JT, Calhoun J, Cobos J. In vitro evaluation of antibiotic diffusion from antibiotic-impregnated biodegradable beads and polymethylmethacrylate beads. Antimicrob Agents Chemother. 1997;41: 415-418.

22. McKee MD, Wild LM, Schemitsch EH, Waddell JP. The use of an antibiotic-impregnated, osteoconductive, bioabsorbable bone substitute in the treatment of infected long bone defects: early results of a prospective trial. J Orthop Trauma. 2002;16:622-627. 
23. Gitelis S, Brebach GT. The treatment of chronic osteomyelitis with a biodegradable antibiotic-impregnated implant. J Orthop Surg. 2002;10:53-60.

24. El-Husseiny M, Patel S, MacFarlane RJ, Haddas FS. Biodegradable antibiotic delivery systems. J Bone Joint Surg. 2011;93:151-157.
25. Huang S, Fu X. Naturally derived materials-based cell and drug delivery systems in skin regeneration. $J$ Control Release. 2010;142: 149-159.

\section{Publish your work in this journal}

The International Journal of Nanomedicine is an international, peerreviewed journal focusing on the application of nanotechnology in diagnostics, therapeutics, and drug delivery systems throughout the biomedical field. This journal is indexed on PubMed Central, MedLine, CAS, SciSearch ${ }^{\circledR}$, Current Contents ${ }^{\circledR} /$ Clinical Medicine,
Journal Citation Reports/Science Edition, EMBase, Scopus and the Elsevier Bibliographic databases. The manuscript management system is completely online and includes a very quick and fair peer-review system, which is all easy to use. Visit http://www.dovepress.com/ testimonials.php to read real quotes from published authors.

Submit your manuscript here: http://www.dovepress.com/international-journal-of-nanomedicine-journal 